\title{
Vibration Attenuation Optimization in a Rod With Different Periodic Piezoelectric Shunting Configurations
}

\author{
Hui Guo, Yaru Zhang, Tao Yuan, Pei Sun Qian, Qian Cheng and Yansong Wang \\ School of Mechanical and Automotive Engineering, Shanghai University of Engineering Science, Shanghai, China. \\ Email: hgsues@163.com
}

\begin{abstract}
(Received 23 November 2020; accepted 13 June 2021)
Wave propagation control in piezoelectric meta-materials has been extensively investigated in recent years due to its significant effects on elastic wave attenuation. In this work, a novel piezoelectric meta-material rod connected to three configurations of shunting circuits is proposed for broad band gaps. The numerical model is constructed to predict the band gap, attenuation constant, and vibration transmission. For larger attenuation within the band gaps, the shunting circuit parameters are optimized with a genetic algorithm. The result shows that the structure with the optimized parameters provides prominent vibration control ability. Both the attenuation constant and the width of the band gaps are enlarged.
\end{abstract}

\section{INTRODUCTION}

Owing to the prominent property of elastic wave propagation in acoustic meta-materials, the prohibition of acoustic or elastic wave propagation in a certain frequency range can be realized. Studies on periodic structures have attracted considerable attention over several decades. ${ }^{1-5}$ Numerous applications may benefit from band gaps for elastic wave propagation, such as cloaking, ${ }^{6}$ acoustic super lenses, ${ }^{7}$ wave guiding, ${ }^{8}$ wave filtering, ${ }^{9,10}$ and vibration attenuation. ${ }^{11}$

Research on acoustic meta-materials has been extended to smart meta-materials, particularly piezoelectric (PZT) shunting circuits having advantages such as light weight, adjustable physical characteristics, and good performance on elastic wave attenuation. This technique was first proposed and exploited to generate broadband wave attenuation in a rod through the unique properties of periodic structures. ${ }^{12}$ Subsequently, a shunted PZT patches array is widely adopted to obtain frequency domain which exhibits significant attenuation of waves and is generally known as band gaps or stop bands. Band gaps are divided into the Bragg-type and the local resonant band gap. The former band gap generation mechanism is related to the impedance mismatch introduced through the use of shunted actuators. ${ }^{13}$ The latter one induces a local resonant band gap with a resonant shunting circuit, which acts as a vibration absorber at the resonance frequency of the circuit. It is easier to achieve tunable local resonant band gaps by simply adjusting the circuit parameters. ${ }^{14-16}$ Various applications are constructed based on the PZT shunt damping that include noise and vibration control in aerospace structures, ${ }^{17}$ automotive components, ${ }^{18}$ turbomachine, and precision electronics. ${ }^{19}$

Driven by the need for broad frequency band gaps, many studies have focused on the design of shunting circuits. More intricate shunting circuits connected to the PZT patches are introduced in the vibration control of beams, ${ }^{20,21}$ and plates, ${ }^{13,22,23}$ including multi-mode resonant shunt, negative capacitive shunt, and synchronized switch shunt. ${ }^{24,25}$ Multimode shunt damping circuits were developed to provide con- trol over multiple structural modes with a single PZT transducer. By placing resistance and inductance (RL) components in parallel with a few similar branches on each PZT patch, ${ }^{20,26}$ these periodic multi-mode shunting PZT arrays can produce multiple local resonant band gaps and obtain a wider range of resonance frequency. Forward was the first scholar to suggest the use of negative capacitance shunts. ${ }^{27}$ A part of the inherent capacitance of the patch can be canceled to significantly enhance the electro-mechanical coupling effect. Therefore, many researchers have illustrated the marked advantages of the negative capacitance shunt to suppress the resonant frequencies of various structures, ${ }^{28-31}$ increase the control bandwidth, and resist perturbations in the structural resonances. ${ }^{32-34}$ Prior results show that negative capacitance is a simple but effective approach for broadening band gaps and increasing attenuation within band gaps.

In the present study, a new PZT meta-material rod connected to resonant shunting circuits with negative capacitance is proposed. Each unit cell includes two independent shunting circuits; one is an RL shunting circuit with capacitance (RL-C), and the other is an RL shunting circuit with negative capacitance (RL-NC). To produce multiple broad band gaps, three topologies for these shunting circuits are presented in Section 2.1. The resonant shunting circuits and negative capacitance are simultaneously utilized to combine the advantages of the two concepts and enhance the two kinds of band gaps. The impedance of the shunting circuit is determined by circuit parameters. The impedance will affect the location and the attenuation amplitude of the band gap. A genetic algorithm (GA) is utilized to optimize the circuit parameters, and the results verify the effectiveness of the optimization. The effect of negative capacitance on the Bragg-type band is investigated.

This paper is organized into five sections including this introduction. The configuration and numerical model of the proposed meta-material with RL-C and RL-NC shunting circuits are presented in section, "Configuration and numerical model". Implementation of negative capacitance is also discussed in this section. The genetic algorithm, direct comparisons before 


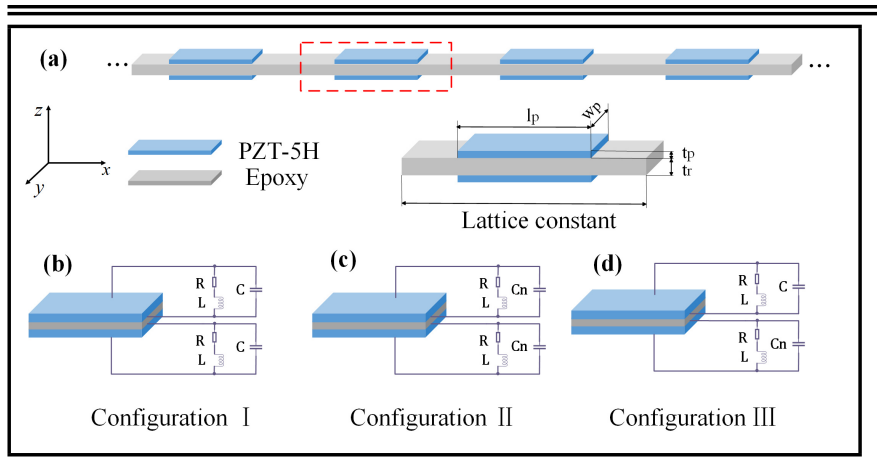

Figure 1. (a) The proposed meta-material with surface-bonded PZT patches; (b) Piezoelectric patch with independent RL-C shunting circuits with Configuration I; (c) Piezoelectric patch with independent RL-NC shunting circuits with Configuration II; (b) Piezoelectric patch with independent RL-C and RLNC shunting circuits with Configuration III.

and after optimization, and analysis are presented in section "Result and discussion". The experimental setup is described along with a detailed overview of practical aspects related to the implementation of the shunting circuits in section "Experimental setup". Finally, conclusions are summarized in section "Conclusion".

\section{CONFIGURATION AND NUMERICAL MODEL}

\subsection{Mathematical Model}

As illustrated in Fig. 1(a), pairs of PZT patches are periodically stuck at the same position on the upper and lower surfaces of the host rod to construct a one-dimensional meta-material along the $x$-axis. A unit cell contains two parts, where the parts with the PZT patches are denoted Segment A, and the other parts are denoted Segment B. Each pair of PZT patches is placed with opposite polarizing directions along the $z$-axis and linked with the independent shunting circuit, consisting of a resistor $R$, inductor $L$ and capacitor $C$ or negative capacitor $C_{n}$ in three topologies, as shown in Fig. 1(b), (c), and (d). These three topologies are used separately in the proposed meta-materials. Configuration I has the upper and lower PZT patches connected to the RL-C circuit, Configuration II has the upper and lower PZT patches connected to the RL-NC circuit, and Configuration III has one PZT patch connected to the RL$\mathrm{C}$ circuit and the other connected to the RL-NC circuit.

It is assumed that all faces of each PZT patch are free of constraints, except the two surfaces along the $x-y$ plane. The PZT patches are only subjected to an electric field along the $z$-axis. Therefore, the piezoelectric equations can be reduced as:

$$
\left[\begin{array}{c}
S_{1} \\
D_{3}
\end{array}\right]=\left[\begin{array}{ll}
s_{11}^{E} & d_{31} \\
d_{31} & \varepsilon_{33}^{T}
\end{array}\right]\left[\begin{array}{l}
T_{1} \\
E_{3}
\end{array}\right]
$$

where 1,2 , and 3 represent coordinates $x, y$, and $z$, respectively. $S_{1}$ and $T_{1}$ are the mechanical strain and stress tensors along the $x$-axis. $D_{3}$ and $E_{3}$ are the electrical displacements and internal electric field in the material. $d_{31}$ is the piezoelectric strain constant. $s_{11}^{E}$ is the compliance coefficient of the PZT patch at constant electric field intensity and $\varepsilon_{33}^{T}$ is the permittivity matrix of the PZT patch at constant stress.

The impedance of each shunting circuit can be expressed as:

$$
Z(s)=\frac{R+\mathrm{Ls}}{1+\mathrm{RCs}+\mathrm{LC} s^{2}} ;
$$

where $R, L$, and $C$ are the resistance, inductance, and capacitance of the shunting circuit, respectively, and $s$ is the Laplace operator.

The current $I$ in the shunting circuit can be written as follows using Ohm's law,

$$
I=-\mathrm{sQ}=\frac{E_{3} h_{p}}{Z(s)}
$$

where $h_{p}$ is the thickness of the PZT patch and $Q$ is the electric charge generated by deformation of the PZT patch.

Assuming the wavelength is long while strain and electrical displacement are equal on each electrode, the charge on the electrodes can be written as:

$$
Q=D_{3} A_{S}
$$

where $A_{S}$ is the surface area of the PZT patch.

Applying Eq. (3) and Eq. (4) in Eq. (1), the relationship between stress and strain can be expressed as

$$
T_{1}=\frac{1}{s_{11}^{E}}\left[1+\frac{s d_{31}^{2} A_{s} Z}{s_{11}^{E} h_{p}\left(1+\mathrm{sZ} C_{p}\right)}\right] S_{1} ;
$$

where $A_{S}$ and $C_{p}$ are the surface area and inherent capacitance of the PZT patch, which can be defined as:

$$
C_{p}^{s}=\frac{\varepsilon_{33}^{S} A_{s}}{h_{p}} .
$$

Under the above assumptions, the effect of shunting circuit is equal to the addition of elastic modulus. The equivalent elastic modulus of the PZT patch with shunting circuit can be written as: mechanical stress divided by strain:

$$
\begin{gathered}
E_{p_{u}}=\frac{1}{s_{11}^{E}}\left[1+\frac{s d_{31}^{2} A_{s} Z_{u}}{h_{p} s_{11}^{E}\left(1+s C_{p}^{s} Z_{u}\right)}\right] ; \\
E_{p_{l}}=\frac{1}{s_{11}^{E}}\left[1+\frac{s d_{31}^{2} A_{s} Z_{l}}{h_{p} s_{11}^{E}\left(1+s C_{p}^{s} Z_{l}\right)}\right] ; \\
E_{A}=\frac{\left(E_{p_{u}}+E_{p_{l}}\right) A_{p}+E_{b} A_{b}}{2 A_{p}+A_{b}}
\end{gathered}
$$

where $A_{p}$ and $A_{b}$ are the cross-sectional areas of the PZT patch and host rod, respectively. $Z_{u}$ and $Z_{l}$ are the impedance of the shunting circuit on the upper and lower PZT patch, severally. $E_{p_{u}}, E_{p_{l}}, E_{A}$, and $E_{b}$ are the elastic modulus of the upper PZT patch, lower PZT patch, Segment A, and host rod, respectively.

\subsection{Dynamic Model}

The transfer matrix can be derived from the governing differential equations and periodic boundary conditions. The influence of the adhesive layer on the structural dynamics is ignored. The longitudinal vibration of each cell is governed by the following differential equation:

$$
\rho \frac{\partial^{2} u}{\partial^{2} t}=\frac{\partial}{\partial x}\left(E \frac{\partial u}{\partial x}\right)
$$

where $\rho, E$, and $u$ are the densities, elastic modulus, displacement of the rod at coordinate $x$, and time $t$, respectively. 
The spatial portion of the general solution to Eq. (8) is:

$$
\begin{aligned}
u_{n, i} & =A_{\mathrm{ni}} \cos \left(\lambda_{i} x_{n, i}\right)+B_{\mathrm{ni}} \sin \left(\lambda_{i} x_{n, i}\right) ; \\
i & =A, \mathrm{~B}
\end{aligned}
$$

where $u_{n, i}$ is the vibration amplitudes, and $A_{\text {ni }}$ and $B_{\text {ni }}$ are undetermined coefficients. $\lambda_{i}$ is the wave number, which is given by:

$$
\lambda_{i}=\sqrt{\frac{\rho_{b} A_{b}+2 \rho_{p} A_{p}}{E_{b} A_{b}+2 E_{p, i} A_{p}} \varpi} ;
$$

where $\rho_{b}, A_{b}, E_{b}$ and $\rho_{p}, A_{p}, E_{p, i}$ are the densities, crosssectional areas, and elastic modulus of the host rod and PZT patches, respectively.

The band gaps can be calculated using the transfer-matrix method and the Bloch theorem for infinitely periodic boundary conditions. The elastic wave Eq. (8) can be transformed into an eigenvalue equation:

$$
\operatorname{det}\left[\mathbf{T}(\varpi)-\mathbf{e}^{\mathrm{ika}} \mathbf{I}\right]=0 ;
$$

where $\mathbf{I}$ is unit matrix, and $k$ is the propagation constant defined as

$$
k=\delta+\mathrm{i} \varepsilon
$$

where the attenuation constant $\delta$ and phase constant $\varepsilon$ describe wave propagation through a periodic structure. For a given frequency $\varpi$, the corresponding propagation constant $k$ can be calculated by solving the above eigenvalue equation. The real part of $k$ is the attenuation constant, it indicates that elastic wave can propagate from one period to the next without attenuation when $\delta=0$. The band gap frequencies of the infinite periodic structure correspond to positive attenuation propagation constant.

The transmission factor in the proposed meta-material can also be calculated with the transfer-matrix method, which signifies the propagation properties of elastic waves in finite structure, and it can be obtained as

$$
H(f)=20 \log \left(\frac{W_{\text {out }}}{W_{\text {in }}}\right)
$$

where $W_{\text {in }}$ and $W_{\text {out }}$ are the vibration amplitudes at the input and output sides. ${ }^{35}$

\subsection{Implementation Capacitance Shunts}

Many researchers have demonstrated the suppression of resonant frequencies of many types of structures using a negative capacitance shunt. The active negative capacitive shunting technique is found to be a simple but effective method to broaden the Bragg band gaps. Hence, the simultaneous use of the resonant shunts and the negative capacitance shunts as studied here combine the advantages of the two concepts and thus provide a wider band gap and stronger vibration attenuation. Negative capacitance acts to "cancel" or "compensate" the capacitance of the PZT material, while the resistance in the negative capacitance shunt can dissipate energy over a greater frequency range. ${ }^{32}$ In this work, negative capacitance has been associated with the resonant circuit to increase the electromechanical coupling coefficient of the system through its distinct

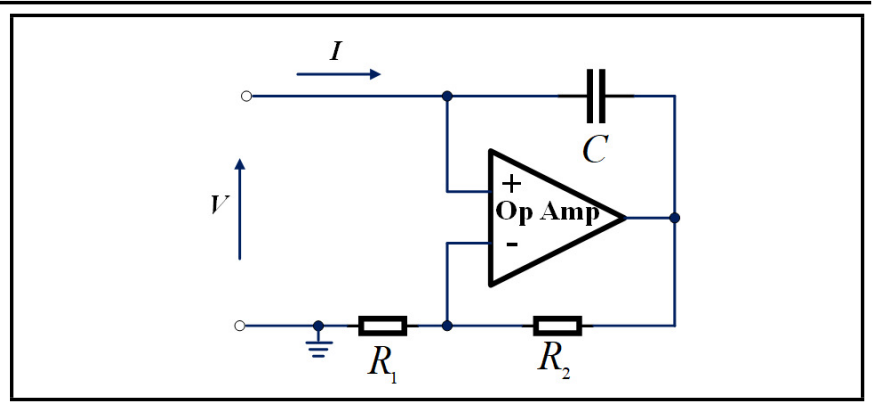

Figure 2. Synthetic negative capacitance circuit.

Table 1. Geometry and material properties of PZT/the host rod (Epoxy).

\begin{tabular}{|l|l|}
\hline Young's modulus of Epoxy & $4.35 \mathrm{GPa}$ \\
Density of Epoxy & $1180\left(\mathrm{~kg} \cdot \mathrm{m}^{-3}\right)$ \\
PZT compliance coefficient $s_{11}^{E}$ & $1.65 \times 10^{-11}\left(\mathrm{~m}^{3} \cdot N^{-1}\right)$ \\
PZT Strain constant $d_{31}$ & $-2.74 \times 10^{-10}\left(\mathrm{C} \cdot \mathrm{m}^{-2}\right)$ \\
PZT Dielectric constant $\varepsilon_{33}^{T}$ & $3.01 \times 10^{-8}\left(\mathrm{~F} \cdot \mathrm{m}^{-1}\right)$ \\
PZT Inherent capacitance & $4.088 \mathrm{e}-8 \mathrm{~F}$ \\
Width of Epoxy $w_{r}$ & $0.02 \mathrm{~m}$ \\
Width of PZT $w_{p}$ & $0.02 \mathrm{~m}$ \\
Thickness of Epoxy $t_{r}$ & $0.01 \mathrm{~m}$ \\
Thickness of PZT $t_{p}$ & $0.0005 \mathrm{~m}$ \\
Length of PZT $l_{p}$ & $0.04 \mathrm{~m}$ \\
Lattice constant & $0.08 \mathrm{~m}$ \\
\hline
\end{tabular}

energy conversion ability. Although a negative capacitor cannot be fabricated, it can be produced with the operational amplifier circuit shown in Fig. 2, which consists of a capacitor, two resistors and an operational amplifier. Eq. (14) shows that any negative capacitance can be obtained by varying the resistors in the synthetic circuit. The equivalent negative capacitance of this circuit is:

$$
C_{n}=-\frac{R_{1}}{R_{2}} \mathrm{C}
$$

\section{RESULTS AND DISCUSSION}

To estimate the vibration control capability of the proposed meta-materials, a one-dimensional meta-material consisting of an epoxy host rod and PZT-5H patches are used to calculate the attenuation constant of the infinite periodic structure and vibration transmission in the finite periodic structure. The geometric parameters and material properties are listed in Table 1.

\subsection{Optimization Scheme}

It has been demonstrated that the resonant frequency of a resonant circuit is strongly related to the center frequency of the local resonant band gap ${ }^{36}$. The local resonant band gap can be affected by any element of the shunting circuit. In particular, the attenuation of the band gap can be affected by the shunt resistance. The width and position of the band gap can be changed by inductance and capacitance. Therefore, the change in effective material properties of the PZT elements can be controlled to tailor the bandwidth and magnitude through shunt design. The corresponding resonant frequency $f$ of the shunting circuits is:

$$
f=\frac{1}{2 \pi \sqrt{L\left(C+C_{p}\right)}} .
$$


As shown in Eq. (15), when determining the position of the band gap, the value of one of the circuit components of the inductance or the capacitor must be determined to obtain the value of the other. The best combination of inductance and capacitance values cannot be directly obtained by Eq. (15). Also the resonant frequency is higher than the attenuation frequency in previous research ${ }^{37}$ In addition, the gap of such a metamaterial is too narrow and has weak attenuation that cannot be used in real situations. It is, therefore, necessary to take measures in the circuits to widen and increase the gap. For these reasons, and to take full advantage of the electromagnetic oscillation and damping dissipation of the shunting circuit, the circuit parameters can be optimized to obtain broad band gaps and achieve bigger attenuation amplitude within band gaps in the three configurations. The GA is applied to optimize the shunting circuit parameters. The optimization objects are inductance $L$, capacitance $C$ and negative capacitance $C_{n}$. In particular, to ensure the stability of the system and predict the attenuation, the negative capacitance should be less than the inherent capacitance, which can also be explained in Eq. (15). The optimization objective is the integrated maximum value of the attenuation constant to maximize the attenuation and the width of the band gap at the same time, which is intended to evaluate the bandwidth and the strength of the attenuation within the band gap. As a natural heuristic stochastic evolutionary algorithm, GA does not require the objective function to be continuously differentiated, and the domain can be assumed arbitrarily. When the optimization desired variables and objectives are determined, the target frequency domain can be set to any desired band gap frequency independently, which denoted as tun ${ }^{-}$and tun ${ }^{+}$to make better use of the tunability of the local resonant band gap through the shunting circuit. First, a parent population is formed by randomly created individuals, which represent the circuit parameters. Secondly, the fitness of each individual is evaluated based on the corresponding objective value calculated by the transfer matrix. A mating pool is constructed by using the roulette wheel algorithm to select individuals from the current population, and then the offspring individuals are worked out through the evolution of selecting, crossing, and mutating which is called an elitist selection strategy. Finally, the fitness of the offspring individuals is evaluated until the terminal condition is satisfied. The flow-chart is shown in Fig. 3 and the objective function can be written as:

$$
\text { Maxmize : } \mathrm{ObjV}=\int_{\text {tun }^{-}}^{\mathrm{tun}^{+}} \text {attenuation constant df. }
$$

\subsection{Band Gaps of the Proposed Meta- Materials}

The corresponding eigenfrequencies $f_{r}$ of the two independent shunting circuit are chosen to be $9 \mathrm{kHz}$ and $10 \mathrm{kHz}$, and the inductances, capacitances, or negative capacitances are calculated using Eq. (15). Detailed information on the circuit parameters is listed in Table 2. Fig. 4 shows the attenuation constant of the meta-material rod with periodic shunted PZT patches arrays in three configurations. Herein, the open circuit case, in which the PZTs are not connected to electronic shunts, is provided as a baseline reference. In parallel, considering resistance is one of the most important elements of effect on the

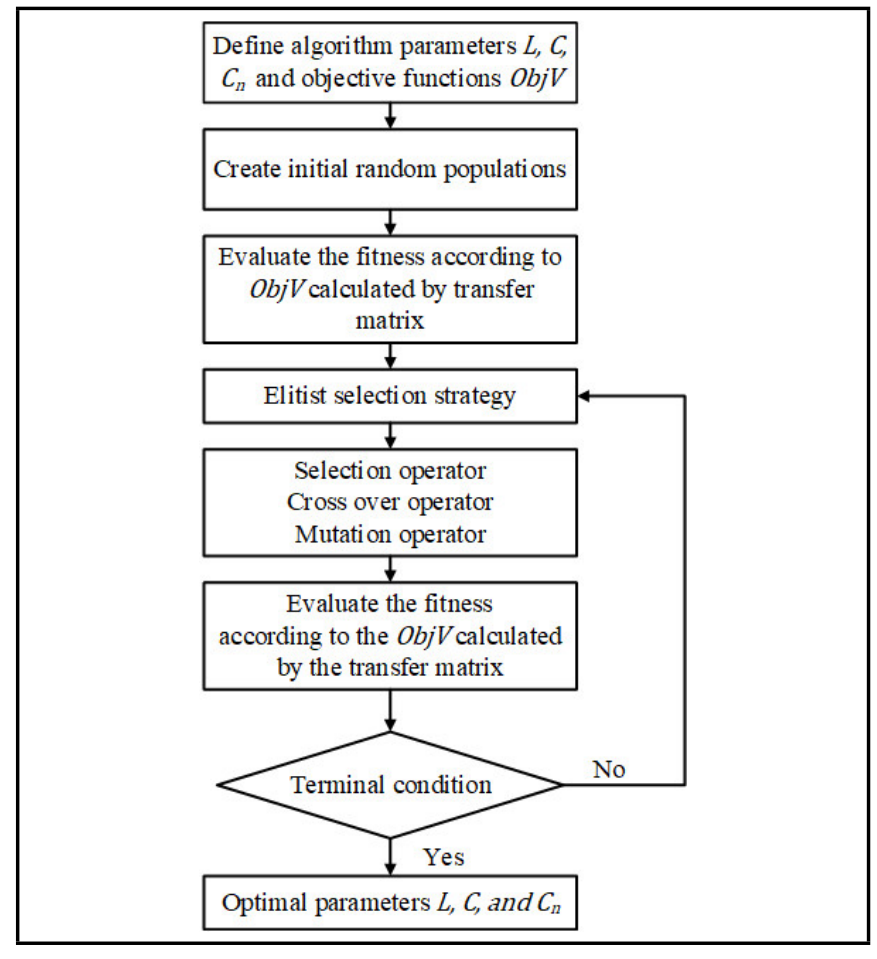

Figure 3. Flow-chart of the optimization scheme.

attenuation factor, the resistance in the three configurations is set to $10 \Omega$ to emphasize the effect of negative capacitance on the band gap. The resonant circuit altered the elastic modulus of the system and the equivalent elastic modulus become zero at a certain frequency, so that elastic waves are localized at the initial cell and unable to propagate in the rod. Bragg-type band gap emerges due to a periodic change in the elastic modulus. It can be seen that a Bragg-type band gap (approximately [10.5 kHz, $16 \mathrm{kHz}]$ ) occurs due to the impedance mismatch between the pure rod and the rod with PZTs in open circuits. In Configuration I, when the upper and lower PZT patches are both linked to the RL-C circuit, employing resonators tuned at adjacent frequencies and displaying slightly overlapping spectra, two local resonant band gaps superimpose to form a wider band gap (approximately $[8 \mathrm{kHz}, 10 \mathrm{kHz}]$ ), named 'rainbow trap'. ${ }^{38}$ The values of inductances are chosen to program the meta-material to realize vibration reduction in a wide and flexible frequency band to have different but successive resonance frequencies. In the three Configurations, the solid line shows the better wave attenuation capability, both in the local resonant band gap and the Bragg-type band gap in Configuration II. Also, the proposed meta-material in Configuration III enhanced the 'rainbow trap' in Configuration I, induces the desired local resonant band gap with greater attenuation and produces a broadened Bragg-type band gap.

Setting the desired frequency from $6 \mathrm{kHz}$ to $12 \mathrm{kHz}$ ensures the local resonant band gaps within this range. Fig. 5 shows the attenuation constant of the proposed meta-material with optimized circuit parameters, which are listed in Table 3. For comparison, the open circuit case is also provided as a reference. In Configuration II and III, a significant optimization effect has been achieved when resonant circuit contains negative capacitance.

The attenuation constant and vibration transmittance of the proposed meta-material with before optimization (solid lines) and after optimization (dashed lines) are shown in Fig 6. We 


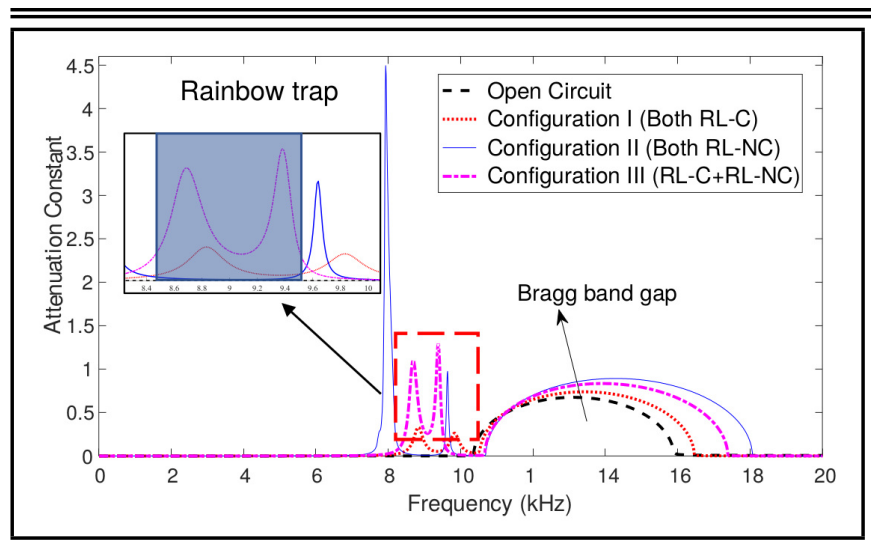

Figure 4. Attenuation constant of the proposed meta-material in three configurations (before optimization).

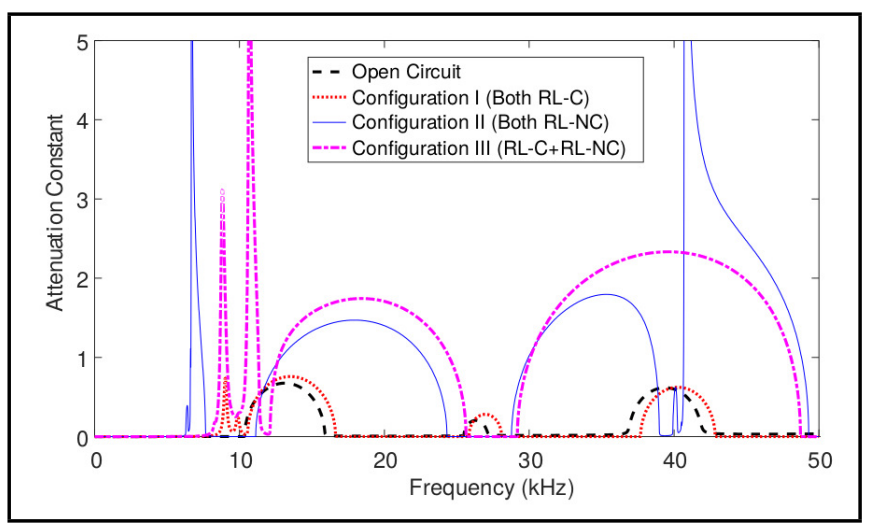

Figure 5. Attenuation constant of the proposed meta-material in three configurations (after optimization).

can see that the optimized circuit parameters generate a higher peak of the attenuation constant while preserving the "rainbow trap" caused by two separate resonant shunt circuits in Fig. 6(a). The attenuation constant in the local resonant band gap is much larger in Configuration II, as shown in Fig. 6(b). Noticeably, the Bragg-type band gap calculated from the optimized circuits has a much bigger attenuation than that calculated from the original circuits in Configurations II and III. Even the width of the Bragg-type band gap is approximately a factor of two larger with the optimized circuit compared to the original circuit. Configuration III shows that the "rainbow trap" generated by the two local resonant shunt circuits has been broadened while significantly magnifying the attenuation constant with the optimized circuit. The asymmetrical configuration showed optimal band gap both in attenuation amplitude and bandwidth. In addition, wave attenuation performances in the proposed structure in these cases have a good agreement with vibration transmittance performance.

\subsection{Effect of the Negative Capacitance}

The above results show that negative capacitance has a great impact on the Bragg-type band gap. The Bragg-type bandwidth in the proposed meta-structure is investigated with different negative capacitance ratios, calculated using Eq. (17). Variations in the Bragg-type bandwidth with a varying negative capacitance ratio are shown in Fig. 7. As the negative capacitance ratio increased, the lower edge of the band gap increased slightly while the upper edge increased significantly, producing a net increment in the width of the Bragg-type band gap as the negative capacitance magnitude approaches the PZT capacitance. The suppression capability of the negative capacitance increases, apparently, as the negative capacitance magnitude becomes closer to the inherent capacitance of the PZT. Theoretically, the absolute optimized value of the negative capacitance connected to the PZT patch is equal to the inherent capacitance of the PZT according to the linear circuit resonant theory. ${ }^{39}$

$$
\lambda=\frac{\left|C_{n}\right|}{C_{p}} .
$$

In Configuration II, the Bragg-type band gaps feature different performances in different cases. For example, the upper and lower RL-NC shunting circuits closed simultaneously and only one of the shunting circuits closed. Thus, the above situation is divided into Case A, B and C. Case A corresponded with the shunting circuit on the upper PZT patch being closed and the shunting circuit on the lower PZT patch being open, while the connections in Case B are reversed. Case C corresponded with the shunting circuit on the upper and lower PZT patches while they are simultaneously closed. The attenuation constants of the proposed meta-materials in different cases of the shunting circuit are shown in Fig. 8. which are calculated using the data in Table 3. Cases A, B, and $\mathrm{C}$ are based on Configuration II. From Fig. 8, the attenuation constant exhibits almost an identical performance. When both shunting circuits are used, the local resonant band gap from $5 \mathrm{kHz}$ to $11 \mathrm{kHz}$ remained, and a wider local resonant band gap from 40 to $50 \mathrm{kHz}$ (the second Bragg-type stop band in Case A and B) is observed in Case C.

\section{EXPERIMENTAL SETUP}

In order to demonstrate the effectiveness of the optimization theory on the proposed structure, the experiment of Configuration II is performed, and the corresponding layout is depicted in Fig. 9. The dimensions and materials employed are the same as those listed in Table 1. Experiments are performed on an epoxy rod of finite extent in order to demonstrate the validity of the proposed approach. The frequency response functions are measured on a rod with 16 surface-bonded PZT patches as shown in Fig 11. The applied force is given by a vibrator (MB), which is fed with white noise with a bandwidth from 0 to $1.2 \mathrm{kHz}$, located at an end of the rod. Two acceleration sensors bonded at both ends of the rod picks up the acceleration responses and gets transmission properties.

Shunted RL circuits require large inductance values based on the numerical results before and after optimization. These values would typically attain dozens of Henries, so that the weight and internal resistance of such inductors would increase significantly, which would make the use of this technique unfeasible and affect the accuracy of the shunted circuit. Synthetic inductors, proposed by Antoniou, are composed of resistance, capacitance, and power amplifiers, as shown in Fig. 10(a). The resulting inductance is given by:

$$
L=\frac{R_{1} R_{3} R_{5}}{R_{2}} C_{4} .
$$

With Eq. (18), any value of the inductance can be changed by varying one resistor of the Antoniou circuit. The resonant shunt and negative capacitance shunt are simultaneously used 
H. Guo, et al.: VIBRATION ATTENUATION OPTIMIZATION IN ROD WITH DIFFERENT PERIODIC PIEZOELECTRIC SHUNTING...

Table 2. Shunting circuit parameters for the upper and lower PZT patches (before optimization).

\begin{tabular}{|l|l|l|l|l|l|l||}
\hline Configuration & \multicolumn{3}{|c|}{ Configuration I } & \multicolumn{2}{c|}{ Configuration II } & \multicolumn{2}{c|}{ Configuration III } \\
\hline Shunting circuit on upper & $L_{u}(H)$ & $C_{u}(F)$ & $L_{u}(H)$ & $C_{u}(F)$ & $L_{u}(H)$ & $C_{u}(F)$ \\
PZT patch & $5 \times 10^{-3}$ & $2.173 \times 10^{-8}$ & $2.88 \times 10^{-2}$ & $-3 \times 10^{-8}$ & $5 \times 10^{-3}$ & $2.173 \times 10^{-8}$ \\
\hline Shunting circuit on lower & $L_{l}(H)$ & $C_{l}(F)$ & $L_{l}(H)$ & $C_{l}(F)$ & $L_{l}(H)$ & $C_{l}(F)$ \\
PZT patch & $5 \times 10^{-3}$ & $0.983 \times 10^{-8}$ & $2.33 \times 10^{-2}$ & $-3 \times 10^{-8}$ & $2.33 \times 10^{-2}$ & $-3 \times 10^{-8}$ \\
\hline
\end{tabular}

Table 3. Shunting circuit parameters for the upper and lower PZT patches (after optimization).

\begin{tabular}{|l|l|l|l|l|l|l||}
\hline Configuration & \multicolumn{2}{|c|}{ Configuration I } & \multicolumn{2}{c|}{ Configuration II } & \multicolumn{2}{c|}{ Configuration III } \\
\hline Shunting circuit on upper & $L_{u}(H)$ & $C_{u}(F)$ & $L_{u}(H)$ & $C_{u}(F)$ & $L_{u}(H)$ & $C_{u}(F)$ \\
PZT patch & $6 \times 10^{-3}$ & $1.002 \times 10^{-8}$ & 0.135 & $-4.065 \times 10^{-8}$ & $4.69 \times 10^{-3}$ & $1.911 \times 10^{-8}$ \\
\hline Shunting circuit on lower & $L_{l}(H)$ & $C_{l}(F)$ & $L_{l}(H)$ & $C_{l}(F)$ & $L_{l}(H)$ & $C_{l}(F)$ \\
PZT patch & $7 \times 10^{-3}$ & $1.026 \times 10^{-8}$ & 0.125 & $-4.087 \times 10^{-8}$ & 0.123 & $-4.076 \times 10^{-8}$ \\
\hline
\end{tabular}
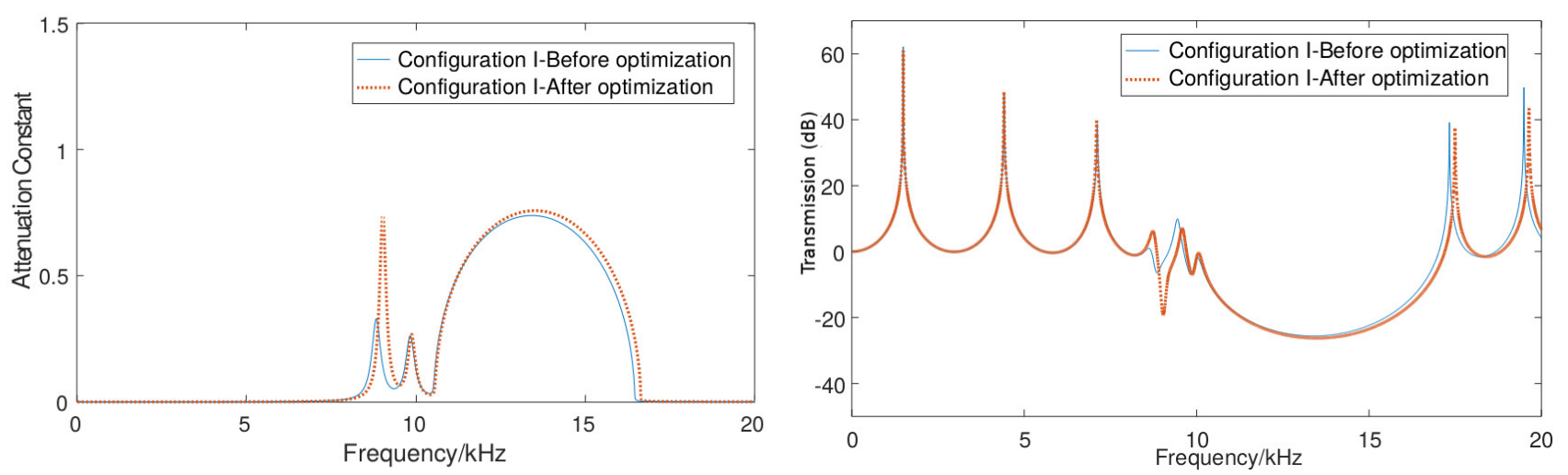

(a)
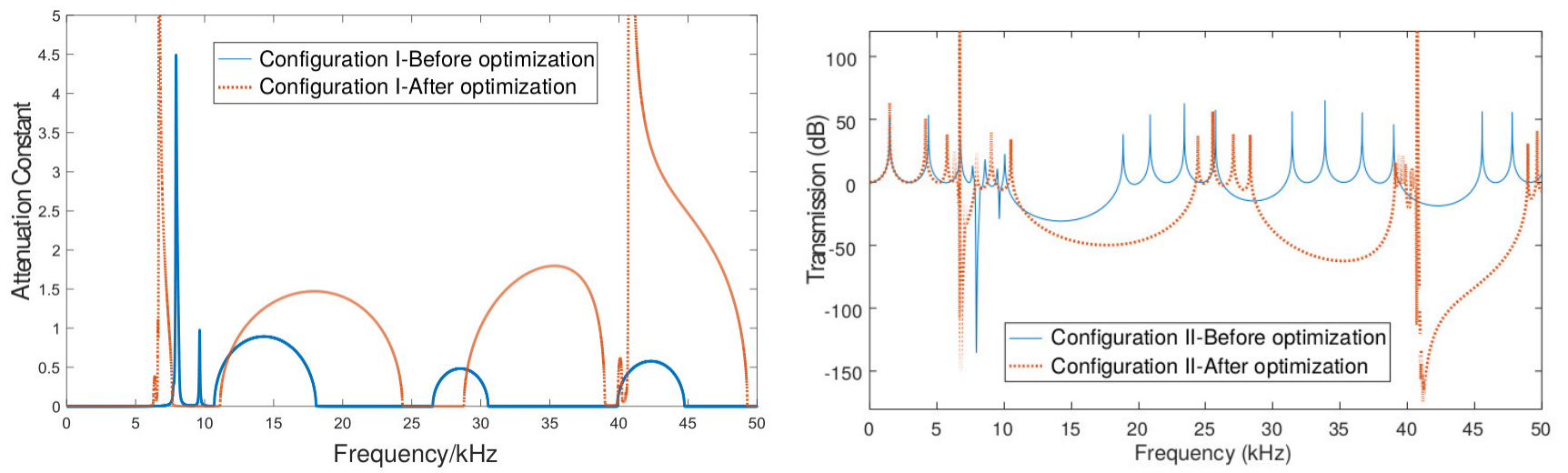

(b)
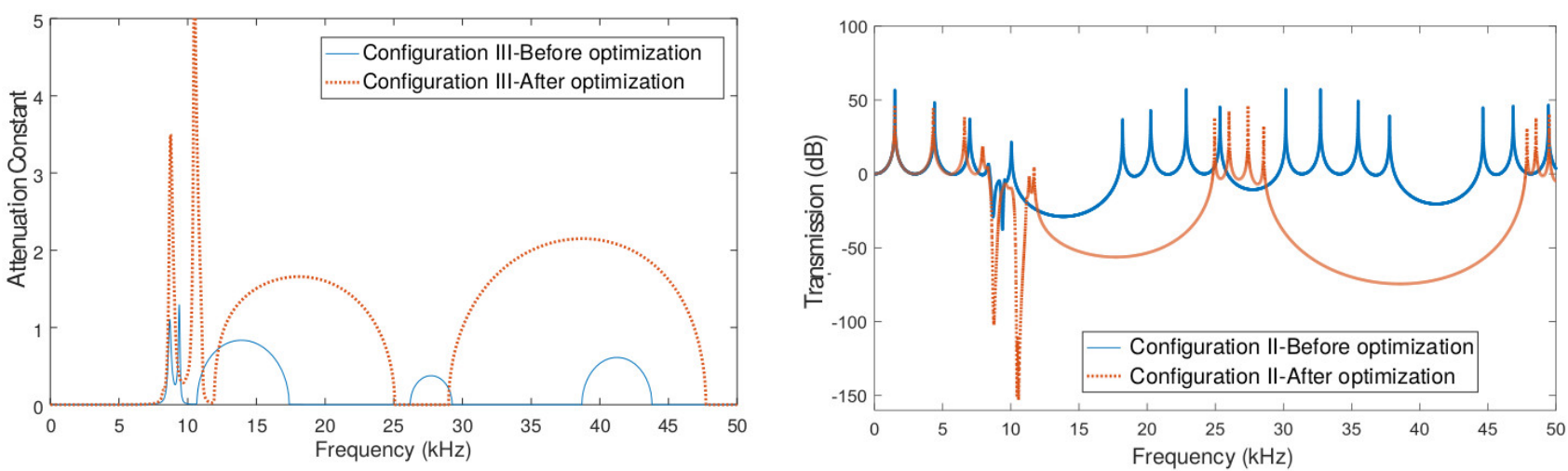

(c)

Figure 6. Attenuation constant and vibration transmittance of the proposed meta-materials in three configurations. (a) Comparison between before and after optimization in Configuration I. (b) Comparison between before and after optimization in Configuration II. (c) Comparison between before and after optimization in Configuration III. 


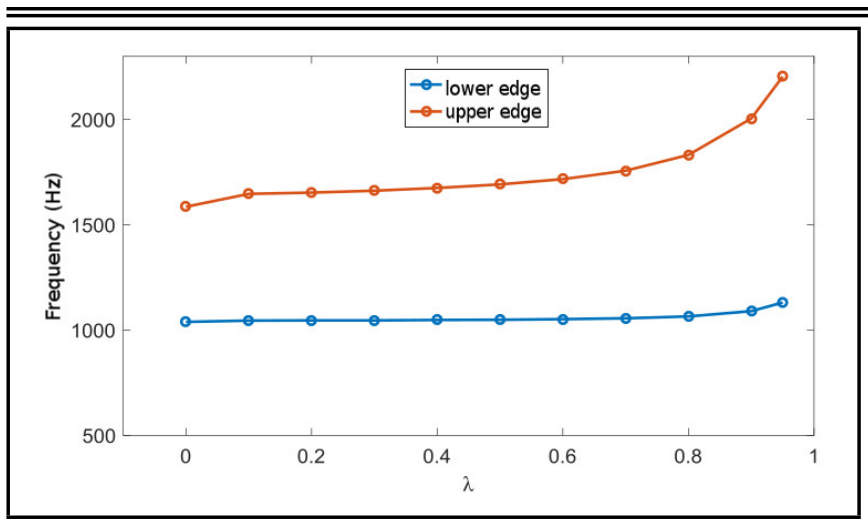

Figure 7. Variations in the Bragg-type bandwidth with changes in negative capacitance ratio $(\lambda)$.

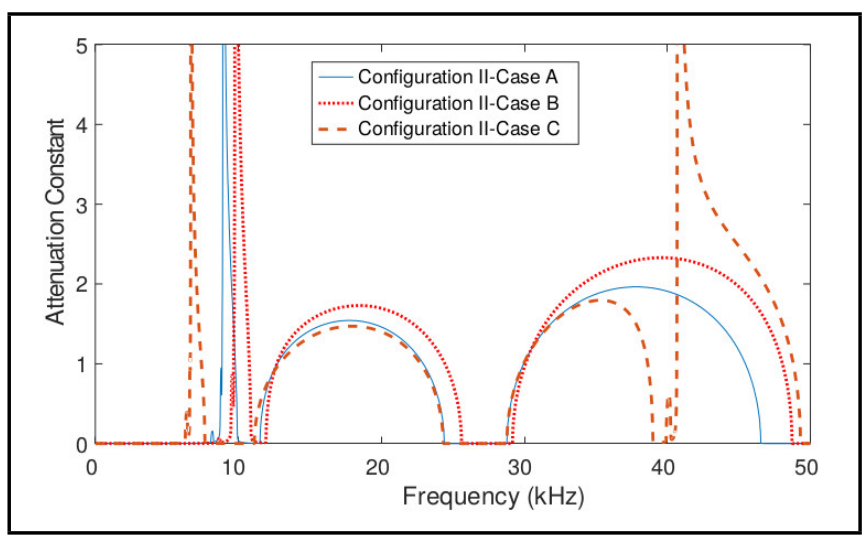

Figure 8. Attenuation constant with open and closed circuits in Configuration II.

in Configuration II, according to the Antoniou circuit and the Synthetic negative capacitance circuit, the Multisim simulation synthesis circuit is shown in Fig. 10(b). Each PZT patch is shunted through a single independent printed circuit board (PCB) on the basis of the simulation synthesis.

Both numerical and experimental results obtained with the shunted PZTs are compared with the short circuit as the reference case. Since RL-NC has a more obvious attenuation effect on the local resonant band gap, the experiment focused on the local resonant band gap. The experimental results of the proposed structure with RL-NC shunts are shown in Fig. 12. Comparing the curve of the short circuit, one can find that the RL-NC shunts produced attenuation in the frequency region of the local resonant band gap $(6500 \sim 8000 \mathrm{~Hz})$ corresponding to the numerical results in Fig. 6(b) on before and after optimization. In particular, an enormous attenuation occurred when RL-NC shunts are optimized by GA. The experimental results match approximately with the results of the theo-

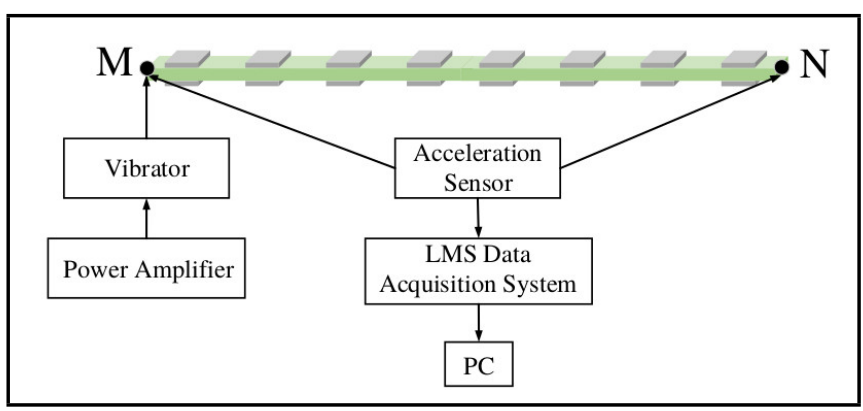

Figure 9. Experimental test scheme diagram.

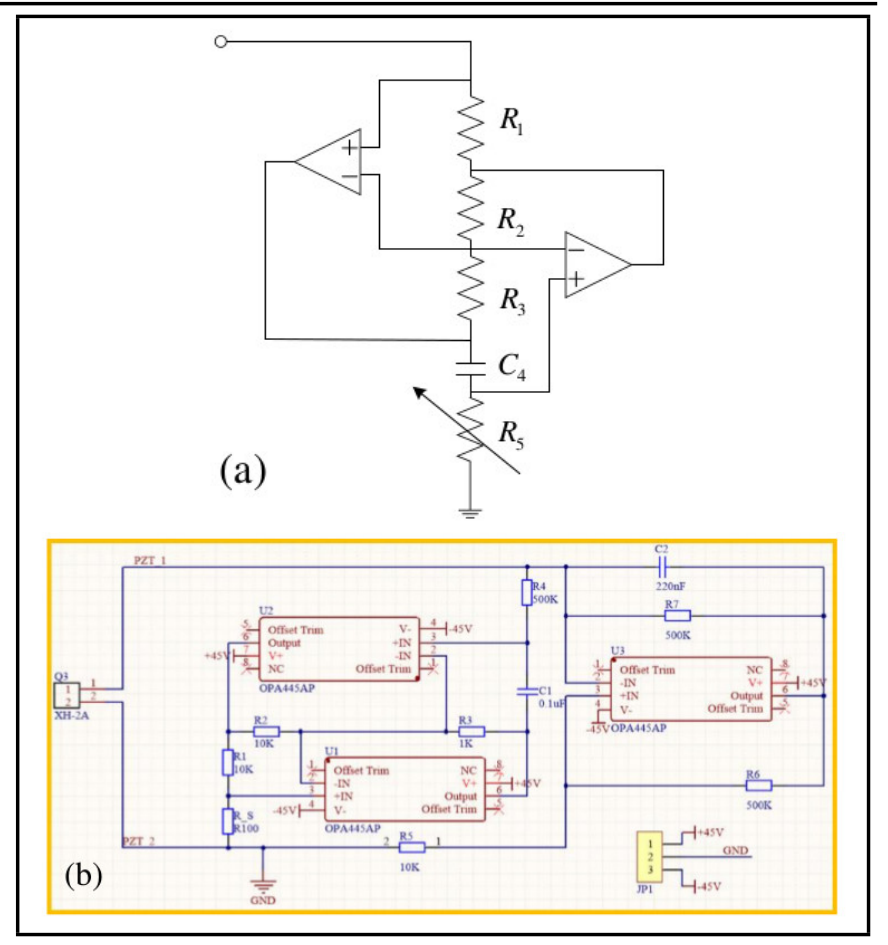

Figure 10. Synthesis circuit (a) Synthetic inductor (b) Multisim simulation synthesis circuit.

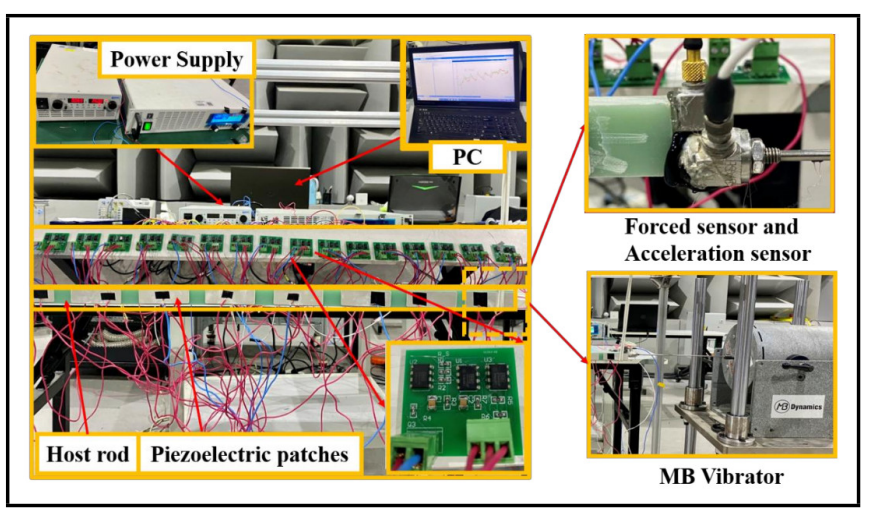

Figure 11. Picture of the experimental site.

retical (Section3.2), thus demonstrating the effects on elastic wave attenuation in the proposed structure and the effectiveness of optimization theory. Although the local resonant band gap frequency obtained from the experiment is a little higher than the numerical results, the deviation of the error may be due to the limited value of the chip resistance and capacitance, which cannot reach the calculated value as accurately as possible. In the shunt circuit, the heat loss cannot be ignored owing to the external power supply. And the existence of the adhesive layer between the PZT patches and rod causes errors. In the following work, research of the local resonant band gap frequency errors will be conducted for further exploration.

\section{CONCLUSION}

An acoustic meta-material structure has been constructed to control the propagation of vibration in a rod with independent RL-C or RL-NC shunting circuits on PZT patches in three configurations. Numerical results show that the two independent shunting circuits can be used to increase band gaps and provide greater attenuation. A GA is applied to optimize the circuit pa- 


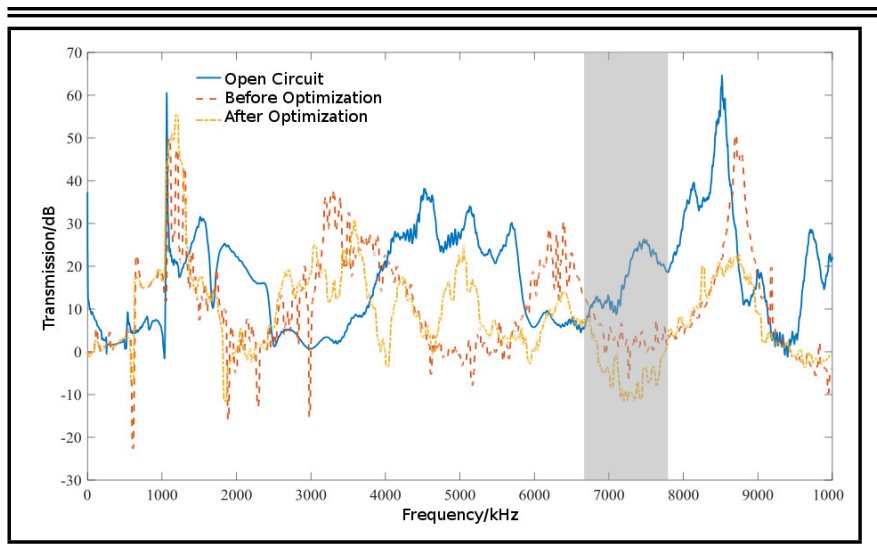

Figure 12. Experimental results of the proposed structure with RL-NC shunts.

rameters, and the optimized circuit can increase the magnitude of the attenuation constant within local resonant band gap and the Bragg band gap.

In Configuration I, a 'rainbow trap' is created by employing resonators tuned at adjacent frequencies, and the optimized circuit parameters generate a much bigger attenuation amplitude without sacrificing attenuation bandwidth. In Configuration II and III, with the negative capacitances added and optimized circuit parameters, a higher peak of the attenuation constant has been achieved. Especially in Configuration III, optimization is more significant for the "rainbow trap". The proposed meta-material and topologies of shunting circuits provide an easy and efficient design for the vibration control. Finally, the experimental results verify the effectiveness of the optimization theory on the proposed structure.

\section{ACKNOWLEDGEMENTS}

This work was supported by the Project of National Natural Science Foundation of China (No. 52172371), and partly supported by the Program for Professor of Special Appointment (Eastern Scholar) at Shanghai Institutions of Higher Learning, Program of Shanghai Academic/Technology Research Leader (No.21XD1401100), and Technical Service Platform for Noise and Vibration Evaluation and Control of New Energy Vehicles (No.18DZ2295900) at Science and Technology Commission of Shanghai Municipality, China.

\section{REFERENCES}

1 Sigalas, M. M and Economou, N. E. Elastic and acoustic wave band structure, Journal of Sound and Vibration, 158(2), 377-382, (1992). https://dx.doi.org/10.1016/0022460X(92)90059-7

2 Banerjee, A. Das, R. and Calius, E. P. Waves in Structured Mediums or Metamaterials: A Review, Archives of Computational Methods in Engineering, 26, 1029-1058, (2018). https://dx.doi.org/10.1007/s11831-018-9268-1

3 Kushwaha, M. Halevi, P. and L. Dobrzynski, Acoustic band structure of periodic elastic composites, Physical Review Letters, 71(13), 2022, (1993). https://dx.doi.org/10.1103/PhysRevLett.71.2022

4 Liu, Z. Zhang, X. and Mao, Y. Locally resonant sonic materials, Science, 289(5485), 1734-1736, (2000). https://dx.doi.org/10.1016/S0921-4526(03)00487-3
5 Martínez-Sala, R. Sancho, J. and Sánchez J, J. Sound attenuation by sculpture, Nature, 378(6554), 241, (1995). https://dx.doi.org/10.1038/378241a0

6 Zhang, S. Xia, C. and Fang, N. Broadband acoustic cloak for ultrasound waves, Physical Review Letters, 106(2), 024301, (2001). https://dx.doi.org/10.1103/PhysRevLett.106.024301

7 Park, J. C. and Lee, K. Acoustic superlens using membranebased metamaterials, Applied Physics Letters, 106(5), 051901, (2015). https://dx.doi.org/10.1063/1.4907634

8 Wang, Y. Wang, T. and Liu, J. Guiding and splitting Lamb waves in coupled-resonator elastic waveguides, Composite Structures, 206, 588-593, (2018). https://dx.doi.org/10.1016/j.compstruct.2018.08.088

9 Zhang, P. and To, A. Broadband wave filtering of bioinspired hierarchical phononic crystal, Applied Physics Letters, 102(12), 121910, (2013). https://dx.doi.org/10.1063/1.4799171

10 Trainiti, G. Xia, Y. and Marconi, J. Time-periodic stiffness modulation in elastic metamaterials for selective wave filtering: Theory and experiment, Physical Review Letters, 122(12), 124301, (2019). https://dx.doi.org/10.1103/PhysRevLett.122.124301

11 Elmadih, W. Chronopoulos, D. and Syam, W. Threedimensional resonating metamaterials for low-frequency vibration attenuation, Scientific report, 9(1), 1-8, (2019). https://dx.doi.org/10.1038/s41598-019-47644-0

12 Thorp, O. Ruzzene, M. and Baz, A. Attenuation and localization of wave propagation in rods with periodic shunted piezoelectric patches", Smart Materials and Structures, 10(5), 979, (2001). https://dx.doi.org/10.1088/0964$1726 / 10 / 5 / 314$

13 Casadei, F. Ruzzene, M. and Dozio, L. Broadband vibration control through periodic arrays of resonant shunts: experimental investigation on plates, Smart Materials and Structures, 19(1), 015002, (2009). https://dx.doi.org/10.1088/0964-1726/19/1/015002

14 Chen, S. Wang, G. and Song, Y. Low-frequency vibration isolation in sandwich plates by piezoelectric shunting arrays, Smart Materials and Structures, 25(12), 125024, (2016). https://dx.doi.org/10.1088/0964$1726 / 25 / 12 / 125024$

15 Thorp, O. Ruzzene, M. and Baz, A. Attenuation of wave propagation in fluid-loaded shells with periodic shunted piezoelectric rings, Smart Materials and Structures, 14(4), 594, (2005). https://dx.doi.org/10.1088/0964$1726 / 14 / 4 / 018$

16 Zhang, H. Wen, J. and Chen, S. Flexural wave bandgaps in phononic metamaterial beam with hybrid shunting circuits, Chinese Physics B, 24(3), 036201, (2015). https://dx.doi.org/10.1088/1674-1056/24/3/036201

17 Sales, P. Rade, A. and Souza, G. De Passive vibration control of flexible spacecraft using shunted piezoelectric transducers, Aerospace Science and Technology, 29(1), 403-412, (2013). https://dx.doi.org/10.1016/j.ast.2013.05.001 
18 Rocha, Teo L. and Dias, M. Improved sound transmission loss in an automotive component using piezoceramic patches and dissipative shunt circuits, Journal of Intelligent Materials Systems and Structures, 26(4), 476-786, (2015). https://dx.doi.org/10.1177/1045389X14529610

19 Silva, M. Venter, G. Experimental results on chatter reduction in turning through embedded piezoelectric material and passive shunt circuits, Mechatronics, 29, 78-85. (2015). https://dx.doi.org/10.1016/j.mechatronics.2015.06.002

20 Airoldi, L. and Ruzzene, M. Wave propagation control in beams through periodic multi-branch shunts, Journal of Intelligent Materials Systems and Structures, 22(14), 1567-1579, (2011). https://dx.doi.org/10.1177/1045389X11408372

21 Zhou, W. Chen, W. and Chen, Z. Actively controllable flexural wave band gaps in beam-type acoustic metamaterials with shunted piezoelectric patches, European Journal of Mechanics - A/Solids, 103807, (2019). https://dx.doi.org/10.1016/j.euromechsol.2019.103807

22 Spadoni, A. Ruzzene, M. and Cunefare, K. Vibration and wave propagation control of plates with periodic arrays of shunted piezoelectric patches, Journal of Intelligent Materials Systems and Structures, 20(8), 979-990, (2009). https://dx.doi.org/10.1177/1045389X08100041

23 Gardonio, P. and Casagrande, D. Shunted piezoelectric patch vibration absorber on two-dimensional thin structures: Tuning considerations, Journal of Sound and Vibration, 395, 26-47, (2017). https://dx.doi.org/10.1016/j.jsv.2017.02.019

24 Yan, L. Bao, B. and Guyomar, D. Periodic structure with interconnected nonlinear electrical networks, Journal of Intelligent Materials Systems and Structures, 28(2), 204-229, (2017). https://dx.doi.org/10.1177/1045389X16649448

25 Bao, B. Guyomar, D. and Lallart, M. Vibration reduction for smart periodic structures via periodic piezoelectric arrays with nonlinear interleavedswitched electronic networks, Mechanical Systems and Signal Processing, 82, 230-259, (2017). https://dx.doi.org/10.1016/j.ymssp.2016.05.021

$26 \mathrm{Wu}$, S. Method for multiple-mode shunt damping of structural vibration using a single PZT transducer. In Smart structures and materials: passive damping and isolation, (Vol. 3327, pp. 159-168), International Society for Optics and Photonics, (1998). https://dx.doi.org/10.1117/12.310680

27 Forward, R. (U.S. Patent), "Electromechanical transducercoupled mechanical structure with negative capacitance compensation circuit", 4, 158, 787, [1979-06-19].

28 Chen, Y. Huang, G. and Sun, C. Band gap control in an active elastic metamaterial with negative capacitance piezoelectric shunting, Journal of Vibration and Acoustics, 136(6), (2014). https://dx.doi.org/10.1115/1.4028378
29 Casadei, F. Beck, B. and Cunefare,K. Vibration control of plates through hybrid configurations of periodic piezoelectric shunts, Journal of Intelligent Materials Systems and Structures, 23(10), 1169-1177, (2012). https://dx.doi.org/10.1177/1045389X12443014

30 Collet, M. Cunefare, K. and Ichchou, M. Wave motion optimization in periodically distributed shunted piezocomposite beam structures, Journal of Intelligent Materials Systems and Structures, 20(7), 787-808, (2009). https://dx.doi.org/10.1177/1045389X08097902

31 Berardengo, M. Manzoni, S. and Thomas, O. Piezoelectric resonant shunt enhancement by negative capacitances: Optimisation, performance and resonance cancellation, Journal of Intelligent Materials Systems and Structures, 29(12), 2581-2606, (2018). https://dx.doi.org/10.1177/1045389X18770874

32 Beck, B. Cunefare, K. and Collet, M. The power output and efficiency of a negative capacitance shunt for vibration control of a flexural system, Smart Materials and Structures, 22(6), 065009, (2013). https://dx.doi.org/10.1088/0964$1726 / 22 / 6 / 065009$

33 Bao, B. and Wang, Q. Elastic wave manipulation in piezoelectric beam meta-structure using electronic negative capacitance dual-adjacent/staggered connections, Composite Structures, 210, 567-580, (2019). https://dx.doi.org/10.1016/j.compstruct.2018.11.053

34 Berardengo, M. Thomas, O. and Giraud-Audine, C. Improved shunt damping with two negative capacitances: an efficient alternative to resonant shunt, Journal of Intelligent Materials Systems and Structures, 28(16), 2222-2238, (2017). https://dx.doi.org/10.1177/1045389X16667556

35 Chen, S. Wave propagation in acoustic metamaterials with resonantly shunted cross-shape piezos, Journal of Intelligent Materials Systems and Structures, 29(13), 2744-2753, (2018). https://dx.doi.org/10.1177/1045389X18778367

36 Wang, G. Chen, S. and Wen, J. Low-frequency locally resonant band gaps induced by arrays of resonant shunts with Antoniou's circuit: experimental investigation on beams, Smart Materials and Structures, 20(1), 015026, (2010). https://dx.doi.org/10.1088/0964-1726/20/1/015026

37 Wang, G. and Chen, S. Large low-frequency vibration attenuation induced by arrays of piezoelectric patches shunted with amplifier-resonator feedback circuits, Smart Materials and Structures, 25,1, (2016). https://dx.doi.org/10.1088/0964-1726/25/1/015004

38 Cardella, D. Celli, P. and Gonella, S. Manipulating waves by distilling frequencies: a tunable shunt-enabled rainbow trap, Smart Materials and Structures, 25,8, (2016). https://dx.doi.org/10.1088/0964-1726/25/8/085017

39 Kim, J. and Jung. Y. Broadband noise reduction of piezoelectric smart panel featuring negative-capacitiveconverter shunt circuit, Journal of the Acoustical Society of America, 120(4), 2017-2025, (2006). https://dx.doi.org/10.1121/1.2259791 